Volume 1(1), 2015, Pages 51-69

ISSN: $2351-8227$

\title{
Pseudo Almost Periodic Solutions for HCNNs with Time-Varying Leakage Delays*
}

\author{
Cemil Tunç ${ }^{\dagger}$
}

\begin{abstract}
In this paper, we consider a class of high-order cellular neural networks (HCNNs) model with time-varying delays in the leakage terms. We give some sufficient conditions which guarantee the exponential stability of pseudo almost periodic solutions for the model. The obtained results complement with some recent ones in the liteature. The technique of proof involves the exponential dichotomy theory and the fixed point theorem. An illustrative example is given with an application.
\end{abstract}

2000 Mathematics Subject Classification. 34C25, 34D40.

Key words and phrases. High-order cellular neural network; pseudo almost periodic solution; exponential stability; time-varying delay; leakage term.

\section{Introduction}

It is well known that retarded functional differential equations describe those systems or processes whose rate of change of state is determined by their past and present states. These equations are frequently encountered as mathematical models of most dynamical process in mechanics, control theory, physics, chemistry, biology, medicine, economics, atomic energy, information theory, etc. For example, it follows from literature that the high-order recurrent neural networks (HCNNs), which include both the CohenGrossberg neural networks and the Hopfield neural networks as special cases, allow high-order interactions between neurons, and therefore have stronger approximation property, faster convergence rate, greater storage capacity, and higher fault tolerance than the traditional first-order neural networks (see Dembo et al. [1]). Hence, in the past years, high-order neural networks have been successfully applied in many areas,

Received April 8, 2015 - Accepted June 28, 2015.

(C)The Author(s) 2015.This article is published with open access by Sidi Mohamed Ben Abdallah University

*This work was supported by.Yuzuncu Yil University, BAP-2014-FEN-B198....

$\dagger$ Corresponding author. Tel.:+(432)2251806 ; fax:+(432)2251802.

Department of Mathematics, Faculty of Sciences, Yüzünc ̈̈ Yll University, 65080, Van-Turkey. 
such as biological science, pattern recognition and optimization (see Psaltis et al.[2], Karayiannis and Venetsanopoulos [3]). In particular, some attention has been paid to the convergence behavior for HRNNs with delays in the leakage terms (see [4-6] and the references therein). Recently, $\mathrm{Xu}$ [7] and Zhang [8] considered the existence and exponential stability of the anti-periodic solutions for the following HCNNs with time-varying delays in the leakage terms:

$$
\begin{aligned}
x_{i}^{\prime}(t)= & -c_{i}(t) x_{i}\left(t-\eta_{i}(t)\right)+\sum_{j=1}^{n} a_{i j}(t) f_{j}\left(x_{j}\left(t-\tau_{i j}(t)\right)\right) \\
& +\sum_{j=1}^{n} \sum_{l=1}^{n} b_{i j l}(t) g_{j}\left(x_{j}\left(t-\alpha_{i j l}(t)\right)\right) g_{l}\left(x_{l}\left(t-\beta_{i j l}(t)\right)\right) \\
& +\sum_{j=1}^{n} \sum_{l=1}^{n} d_{i j l}(t) \int_{0}^{\infty} \sigma_{i j l}(u) h_{j}\left(x_{j}(t-u)\right) d u \int_{0}^{\infty} \nu_{i j l}(u) h_{l}\left(x_{l}(t-u)\right) d u \\
& +I_{i}(t), i=1,2, \cdots, n,
\end{aligned}
$$

in which $n$ corresponds to the number of units in a neural network, $x_{i}(t)$ corresponds to the state vector of the $i$ th unit at the time $t, c_{i}(t)$ represents the rate with which the $i$ th unit will reset its potential to the resting state in isolation when disconnected from the network and external inputs, $a_{i j}(t), b_{i j l}(t)$ and $d_{i j l}(t)$ are the first and second order connection weights of the neural network, respectively, $\eta_{i}(t) \geq 0$ corresponds to the time-varying leakage delays, $\alpha_{i j l}(t) \geq 0, \beta_{i j l}(t) \geq 0$ and $\tau_{i j}(t) \geq 0$ correspond to the transmission delays, $\sigma_{i j l}(u)$ and $\nu_{i j l}(u)$ correspond to the transmission delay kernels, $I_{i}(t)$ denotes the external inputs at time $t, f_{j}, g_{j}$ and $h_{j}$ are the activation functions of signal transmission.

On the other hand, the dynamics of delayed neural networks is mainly affected by the variation of the environment. As mentioned in [9, p87-90] and [10, p77-94], periodically and almost periodically varying environments are the fundamental basis of the theory of natural selection. In contrast with periodical effects, almost periodic effects can be encountered more often, and pseudo almost periodic effects regulate many phenomena excellently. Hence, complex repetitive phenomena can be considered as almost periodic process and an ergodic component. Therefore, the study of the existence and stability of almost periodic solutions and pseudo almost periodic solutions for the first order cellular neural networks (CNNs) models with leakage delays takes great attention (see [11-14] and the references therein).

It should be noted that to the best of our knowledge from the literature, there is no result on the existence of pseudo almost periodic solutions of the HCNNs with timevarying delays in the leakage terms. The aim of this work is to prove the existence and global exponential stability of the pseudo almost periodic solutions for HCNNs (1.1). Our approach is based on the exponential dichotomy theory and contraction mapping fixed point theorem developed in [15].

The initial conditions associated with system (1.1) are of the form

$$
x_{i}(s)=\varphi_{i}(s), x_{i}^{\prime}(s)=\varphi_{i}^{\prime}(s), s \in(-\infty, 0], i=1,2, \cdots, n,
$$


where $\varphi_{i}(\cdot)$ and $\varphi_{i}^{\prime}(\cdot)$ are real-valued bounded and continuous functions defined on $(-\infty, 0]$.

For convenience, we denote by $\mathbb{R}^{n}\left(\mathbb{R}=\mathbb{R}^{1}\right)$ the set of all $n$-dimensional real vectors (real numbers). Let $J=\{1,2, \cdots, n\}$ and $\left\{x_{i}\right\}=\left(x_{1}, x_{2}, \cdots, x_{n}\right)$. For any $\left\{x_{i}\right\} \in \mathbb{R}^{n}$, we let $|x|$ denote the absolute-value vector given by $|x|=\left\{\left|x_{i}\right|\right\}$, and define $\|x\|=$ $\max _{i \in J}\left|x_{i}\right|$. A matrix or vector $A \geq 0$ means that all entries of $A$ are greater than or equal to zero. $A>0$ can be defined similarly. For matrices or vectors $A_{1}$ and $A_{2}, A_{1} \geq A_{2}$ (resp. $\left.A_{1}>A_{2}\right)$ means that $A_{1}-A_{2} \geq 0\left(\right.$ resp. $\left.A_{1}-A_{2}>0\right) . B C\left(\mathbb{R}, \mathbb{R}^{n}\right)$ denotes the set of bounded and continues functions from $\mathbb{R}$ to $\mathbb{R}^{n}$. Note that $\left(B C\left(\mathbb{R}, \mathbb{R}^{n}\right),\|\cdot\|_{\infty}\right)$ is a Banach space, where $\|\cdot\|_{\infty}$ denotes the sup norm $\|f\|_{\infty}:=\sup _{t \in \mathbb{R}}\|f(t)\|$. For $f \in B C(\mathbb{R}, \mathbb{R})$, we set

$$
f^{-}=\inf _{t \in R}|f(t)|, \quad f^{+}=\sup _{t \in R}|f(t)| .
$$

Definition 1.1 (see $[\mathbf{9}, \mathbf{1 0}])$. Let $u(t) \in B C\left(\mathbb{R}, \mathbb{R}^{n}\right)$. $u(t)$ is said to be almost periodic on $\mathbb{R}$ if, for any $\varepsilon>0$, the set $T(u, \varepsilon)=\{\delta:\|u(t+\delta)-u(t)\|<\varepsilon$ for all $t \in \mathbb{R}\}$ is relatively dense, i.e., for any $\varepsilon>0$, it is possible to find a real number $l=l(\varepsilon)>0$ with the property that, for any interval with length $l(\varepsilon)$, there exists a number $\delta=\delta(\varepsilon)$ in this interval such that $\|u(t+\delta)-u(t)\|<\varepsilon$, for all $t \in \mathbb{R}$.

We denote by $A P\left(\mathbb{R}, \mathbb{R}^{n}\right)$ the set of the almost periodic functions from $\mathbb{R}$ to $\mathbb{R}^{n}$. Precisely, define the class of functions $P A P_{0}\left(\mathbb{R}, \mathbb{R}^{n}\right)$ as follows:

$$
\left\{f \in B C\left(\mathbb{R}, \mathbb{R}^{n}\right)\left|\lim _{r \rightarrow+\infty} \frac{1}{2 r} \int_{-r}^{r}\right| f(t) \mid d t=0\right\} .
$$

A function $f \in B C\left(\mathbb{R}, \mathbb{R}^{n}\right)$ is called pseudo almost periodic if it can be expressed as

$$
f=h+\varphi,
$$

where $h \in A P\left(\mathbb{R}, \mathbb{R}^{n}\right)$ and $\varphi \in P A P_{0}\left(\mathbb{R}, \mathbb{R}^{n}\right)$. The collection of such functions will be denoted by $P A P\left(\mathbb{R}, \mathbb{R}^{n}\right)$. The functions $h$ and $\varphi$ in above definition are respectively called the almost periodic component and the ergodic perturbation of the pseudo almost periodic function $f$.

Definition 1.2. Let $x^{*}(t)=\left(x_{1}^{*}(t), x_{2}^{*}(t), \cdots, x_{n}^{*}(t)\right)^{T}$ be the pseudo almost periodic solution of system (1.1). If there exist constants $\alpha>0$ and $M>1$ such that for every solution $x(t)=\left(x_{1}(t), x_{2}(t), \cdots, x_{n}(t)\right)^{T}$ of system (1.1) with any initial value $\varphi(t)=\left(\varphi_{1}(t), \varphi_{2}(t), \cdots, \varphi_{n}(t)\right)^{T}$ satisfying (1.2),

$$
\left\|x(t)-x^{*}(t)\right\|_{1}=\max _{i=1,2, \cdots, n}\left\{\max \left\{\left|x_{i}(t)-x_{i}^{*}(t)\right|,\left|x_{i}^{\prime}(t)-x_{i}^{* \prime}(t)\right|\right\}\right\} \leq M\left\|\varphi-x^{*}\right\|_{0} e^{-\alpha t}, \forall t>0,
$$

where $\left\|\varphi-x^{*}\right\|_{0}=\max \left\{\sup _{t \leq 0} \max _{1 \leq i \leq n}\left|\varphi_{i}(t)-x_{i}^{*}(t)\right|, \sup _{t \leq 0} \max _{1 \leq i \leq n}\left|\varphi_{i}^{\prime}(t)-x_{i}^{*}(t)\right|\right\}$, then $x^{*}(t)$ is said to be globally exponentially stable. 


\section{Preliminary Lemmas}

In this section, we shall first recall some basic definitions, lemmas which are used in what follows.

Throughout this paper, it will be assumed that $c_{i}: \mathbb{R} \rightarrow(0,+\infty)$ is an almost periodic function, $\eta_{i}, \tau_{i j}, \alpha_{i j l}, \beta_{i j l}: \mathbb{R} \rightarrow[0,+\infty)$ and $I_{i}, a_{i j}, b_{i j l}, d_{i j l}: \mathbb{R} \rightarrow \mathbb{R}$ are pseudo almost periodic on $\mathbb{R}$, where $i, j, l \in J$. We also make the following assumptions which will be used later.

$\left(H_{1}\right)$ there exist nonnegative constants $L_{j}^{f}, L_{j}^{g}, L_{j}^{h}, M_{j}^{g}$ and $M_{j}^{h}$ such that

$$
\left|f_{j}(u)-f_{j}(v)\right| \leq L_{j}^{f}|u-v|,\left|g_{j}(u)-g_{j}(v)\right| \leq L_{j}^{g}|u-v|,\left|h_{j}(u)-h_{j}(v)\right| \leq L_{j}^{h}|u-v|,
$$

and

$$
\left|g_{j}(u)\right| \leq M_{j}^{g},\left|h_{j}(u)\right| \leq M_{j}^{h}
$$

where $u, v \in R, j \in J$.

$\left(H_{2}\right)$ For $i, j, l \in J$, the delay kernels $\sigma_{i j l}, \nu_{i j l}:[0, \infty) \rightarrow R$ are continuous, $\left|\sigma_{i j l}(t)\right| e^{\kappa t}$ and $\left|\nu_{i j l}(t)\right| e^{\kappa t}$ are integrable on $[0, \infty)$ for a certain positive constant $\kappa$.

$\left(H_{3}\right)$ For each $i \in J$, there exist constants $\alpha_{i}>0$ and $\xi_{i}>0$, such that

$$
\begin{aligned}
& -c_{i}^{-}+c_{i}^{+} \eta_{i}^{+}+\xi_{i}^{-1} \sum_{j=1}^{n} a_{i j}^{+} L_{j}^{f} \xi_{j}+\xi_{i}^{-1} \sum_{j=1}^{n} \sum_{l=1}^{n} b_{i j l}^{+}\left(M_{j}^{g} L_{l}^{g} \xi_{l}+M_{l}^{g} L_{j}^{g} \xi_{j}\right) \\
& \quad+\xi_{i}^{-1} \sum_{j=1}^{n} \sum_{l=1}^{n} d_{i j l}^{+} \int_{0}^{\infty}\left|\sigma_{i j l}(u)\right| d u \int_{0}^{\infty}\left|\nu_{i j l}(u)\right| d u\left(M_{j}^{h} L_{l}^{h} \xi_{l}+M_{l}^{h} L_{j}^{h} \xi_{j}\right) \\
& \leq-\alpha_{i},
\end{aligned}
$$

and

$$
c_{i}^{-}-\alpha_{i}+c_{i}^{+}\left(1-\frac{\alpha_{i}}{c_{i}^{+}}\right)<1 .
$$

Lemma 2.1 (see $\left[11\right.$, Lemma 2.3 ]). Let $\mathbf{B}=\left\{f \mid f, f^{\prime} \in P A P\left(\mathbb{R}, \mathbb{R}^{n}\right)\right\}$ equipped with the induced norm defined by

$$
\|f\|_{\mathbf{B}}=\max \left\{\|f\|_{\infty},\left\|f^{\prime}\right\|_{\infty}\right\}=\max \left\{\sup _{t \in \mathbb{R}}\|f(t)\|, \sup _{t \in \mathbb{R}}\left\|f^{\prime}(t)\right\|\right\}
$$

then, $B$ is a Banach space.

Lemma 2.2 (see [11, Lemma 3.1 ]. Assume that assumptions $\left(H_{1}\right)$ and $\left(H_{2}\right)$ hold. Then, for $\varphi_{j}, \varphi_{l} \in P A P(\mathbb{R}, \mathbb{R})$,

$$
\int_{0}^{\infty} \sigma_{i j l}(u) h_{j}\left(\xi_{j} \varphi_{j}(t-u)\right) d u, \quad \int_{0}^{\infty} \nu_{i j l}(u) h_{l}\left(\xi_{l} \varphi_{l}(t-u)\right) d u \in P A P(\mathbb{R}, \mathbb{R}), i, j, l \in J .
$$

Definition 2.1 (see $[\mathbf{9 , 1 0}]$ ). Let $x \in \mathbb{R}^{n}$ and $Q(t)$ be an $n \times n$ continuous matrix defined on $\mathbb{R}$. The linear system

$$
x^{\prime}(t)=Q(t) x(t)
$$


is said to admit an exponential dichotomy on $\mathbb{R}$ if there exist positive constants $k, \alpha$, projection $P$ and the fundamental solution matrix $X(t)$ of $(2.2)$ satisfying

$$
\begin{aligned}
& \left\|X(t) P X^{-1}(s)\right\| \leq k e^{-\alpha(t-s)} \quad \text { for } t \geq s \\
& \left\|X(t)(I-P) X^{-1}(s)\right\| \leq k e^{-\alpha(s-t)} \quad \text { for } t \leq s .
\end{aligned}
$$

Lemma 2.3 (see [9]). Assume that $Q(t)$ is an almost periodic matrix function and $g(t) \in P A P\left(\mathbb{R}, \mathbb{R}^{n}\right)$. If the linear system (2.2) admits an exponential dichotomy, then pseudo almost periodic system

$$
x^{\prime}(t)=Q(t) x(t)+g(t)
$$

has a unique pseudo almost periodic solution $x(t)$, and

$$
x(t)=\int_{-\infty}^{t} X(t) P X^{-1}(s) g(s) d s-\int_{t}^{+\infty} X(t)(I-P) X^{-1}(s) g(s) d s .
$$

Lemma 2.4 (see $[\mathbf{9 , 1 0}]$ ). Let $c_{i}(t)$ be an almost periodic function on $\mathbb{R}$ and

$$
M\left[c_{i}\right]=\lim _{T \rightarrow+\infty} \frac{1}{T} \int_{t}^{t+T} c_{i}(s) d s>0, \quad i=1,2, \cdots, n .
$$

Then the linear system

$$
x^{\prime}(t)=\operatorname{diag}\left(-c_{1}(t),-c_{2}(t), \cdots,-c_{n}(t)\right) x(t)
$$

admits an exponential dichotomy on $\mathbb{R}$ (It is worthwhile to mention that the exponential dichotomy in that case is with $P=I$ ).

\section{Existence and uniqueness of pseudo almost periodic solutions}

In this section, we establish sufficient conditions on the existence of pseudo almost periodic solutions of (1.1).

Theorem 3.1. Let $\left(H_{1}\right),\left(H_{2}\right)$ and $\left(H_{3}\right)$ hold. Then, there exists a unique continuously differentiable pseudo almost periodic solution of system (1.1).

Proof. Set

$$
\bar{x}_{i}(t)=\xi_{i}^{-1} x_{i}(t),
$$

then we can transform (1.1) into the following system

$$
\begin{aligned}
\bar{x}_{i}^{\prime}(t)= & -c_{i}(t) \bar{x}_{i}\left(t-\eta_{i}(t)\right)+\xi_{i}^{-1} \sum_{j=1}^{n} a_{i j}(t) f_{j}\left(\xi_{j} \bar{x}_{j}\left(t-\tau_{i j}(t)\right)\right) \\
& +\xi_{i}^{-1} \sum_{j=1}^{n} \sum_{l=1}^{n} b_{i j l}(t) g_{j}\left(\xi_{j} \bar{x}_{j}\left(t-\alpha_{i j l}(t)\right)\right) g_{l}\left(\xi_{l} \bar{x}_{l}\left(t-\beta_{i j l}(t)\right)\right) \\
& +\xi_{i}^{-1} \sum_{j=1}^{n} \sum_{l=1}^{n} d_{i j l}(t) \int_{0}^{\infty} \sigma_{i j l}(u) h_{j}\left(\xi_{j} \bar{x}_{j}(t-u)\right) d u \int_{0}^{\infty} \nu_{i j l}(u) h_{l}\left(\xi_{l} \bar{x}_{l}(t-u)\right) d u \\
& +\xi_{i}^{-1} I_{i}(t)
\end{aligned}
$$




$$
\begin{aligned}
= & -c_{i}(t) \bar{x}_{i}(t)+c_{i}(t) \int_{t-\eta_{i}(t)}^{t} \bar{x}_{i}^{\prime}(s) d s+\xi_{i}^{-1} \sum_{j=1}^{n} a_{i j}(t) f_{j}\left(\xi_{j} \bar{x}_{j}\left(t-\tau_{i j}(t)\right)\right) \\
& +\xi_{i}^{-1} \sum_{j=1}^{n} \sum_{l=1}^{n} b_{i j l}(t) g_{j}\left(\xi_{j} \bar{x}_{j}\left(t-\alpha_{i j l}(t)\right)\right) g_{l}\left(\xi_{l} \bar{x}_{l}\left(t-\beta_{i j l}(t)\right)\right) \\
& +\xi_{i}^{-1} \sum_{j=1}^{n} \sum_{l=1}^{n} d_{i j l}(t) \int_{0}^{\infty} \sigma_{i j l}(u) h_{j}\left(\xi_{j} \bar{x}_{j}(t-u)\right) d u \int_{0}^{\infty} \nu_{i j l}(u) h_{l}\left(\xi_{l} \bar{x}_{l}(t-u)\right) d u \\
& +\xi_{i}^{-1} I_{i}(t), i \in J .
\end{aligned}
$$

Let $\varphi \in \mathbf{B}$. Obviously, the boundedness of $\varphi^{\prime}$ implies that $\varphi_{i}$ is a uniformly continuous function on $\mathbb{R}$ for $i \in J$. Set $\tilde{f}(t, z)=\varphi_{i}(t-z),(i \in J)$. By Theorem 5.3 in [9, p. 58] and Definition 5.7 in [9, p. 59], we can obtain that $\widetilde{f} \in P A P(\mathbb{R} \times \Omega)$ and $\widetilde{f}$ is continuous in $z \in K$ and uniformly in $t \in \mathbb{R}$ for all compact subset $K$ of $\Omega \subset \mathbb{R}$. This, together with $\eta_{i} \in P A P(\mathbb{R}, \mathbb{R})$ and Theorem 5.11 in $[9, \mathrm{p}$. 60], implies that

$$
\varphi_{i}\left(t-\eta_{i}(t)\right) \in P A P(\mathbb{R}, \mathbb{R}), i \in J .
$$

Similarly, we have

$$
\varphi_{j}\left(t-\tau_{i j}(t)\right), \varphi_{j}\left(t-\alpha_{i j l}(t)\right), \varphi_{j}\left(t-\beta_{i j l}(t)\right) \in P A P(\mathbb{R}, \mathbb{R}), i, j, l \in J .
$$

From (3.3), $\left(H_{1}\right)$ and Corollary 5.4 in [9, p. 58], we have

$f_{j}\left(\xi_{j} \varphi_{j}\left(t-\tau_{i j}(t)\right)\right), \quad g_{j}\left(\xi_{j} \varphi_{j}\left(t-\alpha_{i j l}(t)\right)\right), \quad g_{l}\left(\xi_{l} \varphi_{l}\left(t-\beta_{i j l}(t)\right)\right) \in P A P(\mathbb{R}, \mathbb{R}), i, j, l \in J$,

which, together with Lemma 2.2 and the fact that $\varphi_{i}\left(t-\eta_{i}(t)\right) \in P A P(\mathbb{R}, \mathbb{R})$, implies

$$
c_{i}(t) \int_{t-\eta_{i}(t)}^{t} \varphi_{i}^{\prime}(s) d s=c_{i}(t) \varphi_{i}(t)-c_{i}(t) \varphi_{i}\left(t-\eta_{i}(t)\right) \in P A P(\mathbb{R}, \mathbb{R}), \quad i \in J,
$$

and

$$
\begin{aligned}
& \xi_{i}^{-1} \sum_{j=1}^{n} a_{i j}(s) f_{j}\left(\xi_{j} \varphi_{j}\left(t-\tau_{i j}(t)\right)\right) \\
& +\xi_{i}^{-1} \sum_{j=1}^{n} \sum_{l=1}^{n} b_{i j l}(t) g_{j}\left(\xi_{j} \varphi_{j}\left(t-\alpha_{i j l}(t)\right)\right) g_{l}\left(\xi_{l} \varphi_{l}\left(t-\beta_{i j l}(t)\right)\right) \\
& +\xi_{i}^{-1} \sum_{j=1}^{n} \sum_{l=1}^{n} d_{i j l}(t) \int_{0}^{\infty} \sigma_{i j l}(u) h_{j}\left(\xi_{j} \varphi_{j}(t-u)\right) d u \int_{0}^{\infty} \nu_{i j l}(u) h_{l}\left(\xi_{l} \varphi_{l}(t-u)\right) d u \\
& +\xi_{i}^{-1} I_{i}(t) \in P A P(\mathbb{R}, \mathbb{R}), i \in J .
\end{aligned}
$$

For $\varphi \in \mathbf{B}$, we consider the pseudo almost periodic solution $x^{\varphi}(t)$ of the following nonlinear pseudo almost periodic differential equations:

$\bar{x}_{i}^{\prime}(t)=-c_{i}(t) \bar{x}_{i}(t)+c_{i}(t) \int_{t-\eta_{i}(t)}^{t} \varphi_{i}^{\prime}(s) d s+\xi_{i}^{-1} \sum_{j=1}^{n} a_{i j}(t) f_{j}\left(\xi_{j} \varphi_{j}\left(t-\tau_{i j}(t)\right)\right)$ 


$$
\begin{aligned}
& +\xi_{i}^{-1} \sum_{j=1}^{n} \sum_{l=1}^{n} b_{i j l}(t) g_{j}\left(\xi_{j} \varphi_{j}\left(t-\alpha_{i j l}(t)\right)\right) g_{l}\left(\xi_{l} \varphi_{l}\left(t-\beta_{i j l}(t)\right)\right) \\
& +\xi_{i}^{-1} \sum_{j=1}^{n} \sum_{l=1}^{n} d_{i j l}(t) \int_{0}^{\infty} \sigma_{i j l}(u) h_{j}\left(\xi_{j} \varphi_{j}(t-u)\right) d u \int_{0}^{\infty} \nu_{i j l}(u) h_{l}\left(\xi_{l} \varphi_{l}(t-u)\right) d u \\
& +\xi_{i}^{-1} I_{i}(t), i=1,2, \cdots, n .
\end{aligned}
$$

Then, notice that $M\left[c_{i}\right]>0, i=1,2, \cdots, n$, it follows from Lemma 2.4 that the linear system

$$
\bar{x}_{i}^{\prime}(t)=-c_{i}(t) \bar{x}_{i}(t), i \in J
$$

admits an exponential dichotomy on $R$. Thus, by (3.5), (3.6) and Lemma 2.3, we obtain that the system (3.7) has exactly one pseudo almost periodic solution:

$$
\begin{aligned}
x^{\varphi}(t)= & \left\{x_{i}^{\varphi}(t)\right\} \\
= & \left\{\int _ { - \infty } ^ { t } e ^ { - \int _ { s } ^ { t } c _ { i } ( u ) d u } \left[c_{i}(s) \int_{s-\eta_{i}(s)}^{s} \varphi_{i}^{\prime}(u) d u+\xi_{i}^{-1} \sum_{j=1}^{n} a_{i j}(s) f_{j}\left(\xi_{j} \varphi_{j}\left(s-\tau_{i j}(s)\right)\right)\right.\right. \\
& +\xi_{i}^{-1} \sum_{j=1}^{n} \sum_{l=1}^{n} b_{i j l}(s) g_{j}\left(\xi_{j} \varphi_{j}\left(s-\alpha_{i j l}(s)\right)\right) g_{l}\left(\xi_{l} \varphi_{l}\left(s-\beta_{i j l}(s)\right)\right) \\
& +\xi_{i}^{-1} \sum_{j=1}^{n} \sum_{l=1}^{n} d_{i j l}(s) \int_{0}^{\infty} \sigma_{i j l}(u) h_{j}\left(\xi_{j} \varphi_{j}(s-u)\right) d u \int_{0}^{\infty} \nu_{i j l}(u) h_{l}\left(\xi_{l} \varphi_{l}(s-u)\right) d u \\
& \left.\left.+\xi_{i}^{-1} I_{i}(s)\right] d s\right\} .
\end{aligned}
$$

Let

$$
\begin{aligned}
F_{i}(t)= & c_{i}(t) \int_{t-\eta_{i}(t)}^{t} \varphi_{i}^{\prime}(u) d u+\xi_{i}^{-1} \sum_{j=1}^{n} a_{i j}(t) f_{j}\left(\xi_{j} \varphi_{j}\left(t-\tau_{i j}(t)\right)\right) \\
& +\xi_{i}^{-1} \sum_{j=1}^{n} \sum_{l=1}^{n} b_{i j l}(t) g_{j}\left(\xi_{j} \varphi_{j}\left(t-\alpha_{i j l}(t)\right)\right) g_{l}\left(\xi_{l} \varphi_{l}\left(t-\beta_{i j l}(t)\right)\right) \\
& +\xi_{i}^{-1} \sum_{j=1}^{n} \sum_{l=1}^{n} d_{i j l}(t) \int_{0}^{\infty} \sigma_{i j l}(u) h_{j}\left(\xi_{j} \varphi_{j}(t-u)\right) d u \int_{0}^{\infty} \nu_{i j l}(u) h_{l}\left(\xi_{l} \varphi_{l}(t-u)\right) d u \\
& +\xi_{i}^{-1} I_{i}(t), i \in J .
\end{aligned}
$$

Then, $\left\{F_{i}\right\} \in P A P\left(\mathbb{R}, \mathbb{R}^{n}\right)$, and (3.9) implies that

$$
\left\{\int_{-\infty}^{t} e^{-\int_{s}^{t} c_{i}(u) d u} F_{i}(s) d s\right\} \in P A P\left(\mathbb{R}, \mathbb{R}^{n}\right) .
$$

From (3.5), (3.6) and (3.10), we get

$$
\left(x^{\varphi}(t)\right)^{\prime}=\left\{\left(x_{i}^{\varphi}(t)\right)^{\prime}\right\}
$$




$$
=\left\{F_{i}(t)-c_{i}(t) \int_{-\infty}^{t} e^{-\int_{s}^{t} c_{i}(u) d u} F_{i}(s) d s\right\} \in P A P\left(\mathbb{R}, \mathbb{R}^{n}\right) .
$$

Thus, $x^{\varphi} \in \mathbf{B}$.

Now, we define a mapping $T: \mathbf{B} \rightarrow \mathbf{B}$ by setting

$$
(T \varphi)(t)=x^{\varphi}(t), \quad \forall \varphi \in \mathbf{B} .
$$

We next prove that the mapping $T$ is a contraction mapping of the $\mathbf{B}$. In fact, in view of (3.9), $\left(H_{1}\right)$ and $\left(H_{3}\right)$, for $\varphi, \psi \in \mathbf{B}$, we have

$$
\begin{aligned}
& \left\{\left|((T \varphi)(t)-(T \psi)(t))_{i}\right|\right\} \\
= & \left\{\mid \int_{-\infty}^{t} e^{-\int_{s}^{t} c_{i}(u) d u}\left\{c_{i}(s) \int_{s-\eta_{i}(s)}^{s}\left(\varphi_{i}^{\prime}(u)-\psi_{i}^{\prime}(u)\right) d u\right.\right. \\
& +\xi_{i}^{-1} \sum_{j=1}^{n} a_{i j}(s)\left(f_{j}\left(\xi_{j} \varphi_{j}\left(s-\tau_{i j}(s)\right)\right)-f_{j}\left(\xi_{j} \psi_{j}\left(s-\tau_{i j}(s)\right)\right)\right) \\
& +\xi_{i}^{-1} \sum_{j=1}^{n} \sum_{l=1}^{n} b_{i j l}(s)\left[\left(g_{j}\left(\xi_{j} \varphi_{j}\left(s-\alpha_{i j l}(s)\right)\right) g_{l}\left(\xi_{l} \varphi_{l}\left(s-\beta_{i j l}(s)\right)\right)\right.\right. \\
& \left.-g_{j}\left(\xi_{j} \varphi_{j}\left(s-\alpha_{i j l}(s)\right)\right) g_{l}\left(\xi_{l} \psi_{l}\left(s-\beta_{i j l}(s)\right)\right)\right) \\
& +\left(g_{j}\left(\xi_{j} \varphi_{j}\left(s-\alpha_{i j l}(s)\right)\right) g_{l}\left(\xi_{l} \psi_{l}\left(s-\beta_{i j l}(s)\right)\right)\right. \\
& \left.\left.-g_{j}\left(\xi_{j} \psi_{j}\left(s-\alpha_{i j l}(s)\right)\right) g_{l}\left(\xi_{l} \psi_{l}\left(s-\beta_{i j l}(s)\right)\right)\right)\right] \\
& +\xi_{i}^{-1} \sum_{j=1}^{n} \sum_{l=1}^{n} d_{i j l}(s)\left[\left(\int_{0}^{\infty} \sigma_{i j l}(u) h_{j}\left(\xi_{j} \varphi_{j}(s-u)\right) d u \int_{0}^{\infty} \nu_{i j l}(u) h_{l}\left(\xi_{l} \varphi_{l}(s-u)\right) d u\right.\right. \\
& \left.-\int_{0}^{\infty} \sigma_{i j l}(u) h_{j}\left(\xi_{j} \varphi_{j}(s-u)\right) d u \int_{0}^{\infty} \nu_{i j l}(u) h_{l}\left(\xi_{l} \psi_{l}(s-u)\right) d u\right) \\
& +\left(\int_{0}^{\infty} \sigma_{i j l}(u) h_{j}\left(\xi_{j} \varphi_{j}(s-u)\right) d u \int_{0}^{\infty} \nu_{i j l}(u) h_{l}\left(\xi_{l} \psi_{l}(s-u)\right) d u\right. \\
& \left.+M_{l}^{g} L_{j}^{g} \xi_{j}\left|\varphi_{j}\left(s-\alpha_{i j l}(s)\right)-\psi_{j}\left(s-\alpha_{i j l}(s)\right)\right|\right] \\
& \left.\left.\left.\left.+\int_{0}^{\infty} \sigma_{i j l}(u) h_{j}\left(\xi_{j} \psi_{j}(s-u)\right) d u \int_{0}^{\infty} \nu_{i j l}(u) h_{l}\left(\xi_{l} \psi_{l}(s-u)\right) d u\right)\right]\right\} d s \mid\right\} \\
& \left\{\int _ { - \infty } ^ { t } e ^ { - \int _ { s } ^ { t } c _ { i } ( u ) d u } \left[c_{i j}(s) \int_{s-\eta_{i}(s)}^{s}\left|\varphi_{i}^{\prime}(u)-\psi_{i}^{\prime}(u)\right| d u\right.\right. \\
& \\
&
\end{aligned}
$$




$$
\begin{aligned}
& +\xi_{i}^{-1} \sum_{j=1}^{n} \sum_{l=1}^{n}\left|d_{i j l}(s)\right|\left[\int_{0}^{\infty}\left|\sigma_{i j l}(u)\right| d u M_{j}^{h} \int_{0}^{\infty}\left|\nu_{i j l}(u)\right| L_{l}^{h} \xi_{l} \mid \varphi_{l}(s-u)\right)-\psi_{l}(s-u) \mid d u \\
& \left.\left.\left.+\int_{0}^{\infty}\left|\sigma_{i j l}(u)\right| L_{j}^{h} \xi_{j}\left|\varphi_{j}(s-u)-\psi_{j}(s-u)\right| d u \int_{0}^{\infty}\left|\nu_{i j l}(u)\right| d u M_{l}^{h}\right]\right\} d s\right\} \\
\leq & \left\{\int _ { - \infty } ^ { t } e ^ { - \int _ { s } ^ { t } c _ { i } ( u ) d u } \left[c_{i}^{+} \eta_{i}^{+}+\xi_{i}^{-1} \sum_{j=1}^{n} a_{i j}^{+} L_{j}^{f} \xi_{j}\right.\right. \\
& +\xi_{i}^{-1} \sum_{j=1}^{n} \sum_{l=1}^{n} b_{i j l}^{+}\left(M_{j}^{g} L_{l}^{g} \xi_{l}+M_{l}^{g} L_{j}^{g} \xi_{j}\right) \\
& \left.\left.\left.+\xi_{i}^{-1} \sum_{j=1}^{n} \sum_{l=1}^{n} d_{i j l}^{+} \int_{0}^{\infty}\left|\sigma_{i j l}(u)\right| d u \int_{0}^{\infty}\left|\nu_{i j l}(u)\right| d u\left(M_{j}^{h} L_{l}^{h} \xi_{l}+M_{l}^{h} L_{j}^{h} \xi_{j}\right)\right] d s\left\|_{\varphi}-\psi\right\|_{\mathbf{B}}\right)\right\} \\
\leq & \left\{\int_{-\infty}^{t} e^{-\int_{s}^{t} c_{i}(u) d u}\left(c_{i}(s)-\alpha_{i}\right) d s\right\}\|\varphi-\psi\|_{\mathbf{B}} \\
\leq & \left\{\int_{-\infty}^{t} e^{-\int_{s}^{t} c_{i}(u) d u} d\left(-\int_{s}^{t} c_{i}(u) d u\right)-\alpha_{i} \int_{-\infty}^{t} e^{-\int_{s}^{t} c_{i}(u) d u} d s\right\}\|\varphi-\psi\|_{\mathbf{B}} \\
\leq & \left\{1-\alpha_{i} \int_{-\infty}^{t} e^{-\int_{s}^{t} c_{i}^{+} d u} d s\right\}\|\varphi-\psi\|_{\mathbf{B}} \\
\leq & \left\{1-\frac{\alpha_{i}}{c_{i}^{+}}\right\}^{\|}\left\|^{-} \psi\right\|_{\mathbf{B}},
\end{aligned}
$$

and

$$
\begin{aligned}
& \left\{\left|\left((T \varphi)^{\prime}(t)-(T \psi)^{\prime}(t)\right)_{i}\right|\right\} \\
= & \left\{\mid\left[c_{i}(t) \int_{t-\eta_{1}(t)}^{t}\left(\varphi_{i}^{\prime}(u)-\psi_{i}^{\prime}(u)\right) d u\right.\right. \\
& +\xi_{i}^{-1} \sum_{j=1}^{n} a_{i j}(t)\left(f_{j}\left(\xi_{j} \varphi_{j}\left(t-\tau_{i j}(t)\right)\right)-f_{j}\left(\xi_{j} \psi_{j}\left(t-\tau_{i j}(t)\right)\right)\right) \\
& +\xi_{i}^{-1} \sum_{j=1}^{n} \sum_{l=1}^{n} b_{i j l}(t)\left(g_{j}\left(\xi_{j} \varphi_{j}\left(t-\alpha_{i j l}(t)\right)\right) g_{l}\left(\xi_{l} \varphi_{l}\left(t-\beta_{i j l}(t)\right)\right)\right. \\
& \left.-g_{j}\left(\xi_{j} \psi_{j}\left(t-\alpha_{i j l}(t)\right)\right) g_{l}\left(\xi_{l} \psi_{l}\left(t-\beta_{i j l}(t)\right)\right)\right) \\
& +\xi_{i}^{-1} \sum_{j=1}^{n} \sum_{l=1}^{n} d_{i j l}(t)\left(\int_{0}^{\infty} \sigma_{i j l}(u) h_{j}\left(\xi_{j} \varphi_{j}(t-u)\right) d u \int_{0}^{\infty} \nu_{i j l}(u) h_{l}\left(\xi_{l} \varphi_{l}(t-u)\right) d u\right. \\
& \left.\left.-\int_{0}^{\infty} \sigma_{i j l}(u) h_{j}\left(\xi_{j} \psi_{j}(t-u)\right) d u \int_{0}^{\infty} \nu_{i j l}(u) h_{l}\left(\xi_{l} \psi_{l}(t-u)\right) d u\right)\right] \\
& -c_{i}(t) \int_{-\infty}^{t} e^{-\int_{s}^{t} c_{i}(u) d u}\left[c_{i}(s) \int_{s-\eta_{i}(s)}^{s}\left(\varphi_{i}^{\prime}(u)-\psi_{i}^{\prime}(u)\right) d u\right.
\end{aligned}
$$




$$
\begin{aligned}
& +\xi_{i}^{-1} \sum_{j=1}^{n} a_{i j}(s)\left(f_{j}\left(\xi_{j} \varphi_{j}\left(s-\tau_{i j}(s)\right)\right)-f_{j}\left(\xi_{j} \psi_{j}\left(s-\tau_{i j}(s)\right)\right)\right) \\
& +\xi_{i}^{-1} \sum_{j=1}^{n} \sum_{l=1}^{n} b_{i j l}(s)\left(g_{j}\left(\xi_{j} \varphi_{j}\left(s-\alpha_{i j l}(s)\right)\right) g_{l}\left(\xi_{l} \varphi_{l}\left(s-\beta_{i j l}(s)\right)\right)\right. \\
& \left.-g_{j}\left(\xi_{j} \psi_{j}\left(s-\alpha_{i j l}(s)\right)\right) g_{l}\left(\xi_{l} \psi_{l}\left(s-\beta_{i j l}(s)\right)\right)\right) \\
& +\xi_{i}^{-1} \sum_{j=1}^{n} \sum_{l=1}^{n} d_{i j l}(s)\left(\int_{0}^{\infty} \sigma_{i j l}(u) h_{j}\left(\xi_{j} \varphi_{j}(s-u)\right) d u \int_{0}^{\infty} \nu_{i j l}(u) h_{l}\left(\xi_{l} \varphi_{l}(s-u)\right) d u\right. \\
& \left.\left.\left.-\int_{0}^{\infty} \sigma_{i j l}(u) h_{j}\left(\xi_{j} \psi_{j}(s-u)\right) d u \int_{0}^{\infty} \nu_{i j l}(u) h_{l}\left(\xi_{l} \psi_{l}(s-u)\right) d u\right)\right] d s \mid\right\} \\
& \leq\left\{\left[c_{i}^{+} \eta_{i}^{+}+\xi_{i}^{-1} \sum_{j=1}^{n} a_{i j}^{+} L_{j}^{f} \xi_{j}\right.\right. \\
& +\xi_{i}^{-1} \sum_{j=1}^{n} \sum_{l=1}^{n} b_{i j l}^{+}\left(M_{j}^{g} L_{l}^{g} \xi_{l}+M_{l}^{g} L_{j}^{g} \xi_{j}\right) \\
& \left.+\xi_{i}^{-1} \sum_{j=1}^{n} \sum_{l=1}^{n} d_{i j l}^{+} \int_{0}^{\infty}\left|\sigma_{i j l}(u)\right| d u \int_{0}^{\infty}\left|\nu_{i j l}(u)\right| d u\left(M_{j}^{h} L_{l}^{h} \xi_{l}+M_{l}^{h} L_{j}^{h} \xi_{j}\right)\right] d s\|\varphi-\psi\|_{\mathbf{B}} \\
& +c_{i}^{+} \int_{-\infty}^{t} e^{-\int_{s}^{t} c_{i}(u) d u}\left[c_{i}^{+} \eta_{i}^{+}+\xi_{i}^{-1} \sum_{j=1}^{n} a_{i j}^{+} L_{j}^{f} \xi_{j}\right. \\
& +\xi_{i}^{-1} \sum_{j=1}^{n} \sum_{l=1}^{n} b_{i j l}^{+}\left(M_{j}^{g} L_{l}^{g} \xi_{l}+M_{l}^{g} L_{j}^{g} \xi_{j}\right) \\
& \left.\left.+\xi_{i}^{-1} \sum_{j=1}^{n} \sum_{l=1}^{n} d_{i j l}^{+} \int_{0}^{\infty}\left|\sigma_{i j l}(u)\right| d u \int_{0}^{\infty}\left|\nu_{i j l}(u)\right| d u\left(M_{j}^{h} L_{l}^{h} \xi_{l}+M_{l}^{h} L_{j}^{h} \xi_{j}\right)\right] d s\right\}\|\varphi-\psi\|_{\mathbf{B}} \\
& \leq\left\{c_{i}^{-}-\alpha_{i}+c_{i}^{+}\left(1-\frac{\alpha_{i}}{c_{i}^{+}}\right)\right\}\|\varphi-\psi\|_{\mathbf{B}} .
\end{aligned}
$$

From $\left(H_{3}\right)$, we have

$$
0<1-\frac{\alpha_{i}}{c_{i}^{+}}<1
$$

and

$$
K=\max \left\{\max _{1 \leq i \leq n}\left\{1-\frac{\alpha_{i}}{c_{i}^{+}}\right\}, \quad \max _{1 \leq i \leq n}\left\{c_{i}^{-}-\alpha_{i}+c_{i}^{+}\left(1-\frac{\alpha_{i}}{c_{i}^{+}}\right)\right\}<1,\right.
$$

which, together with (3.12) and (3.13), yield

$$
\|T \varphi-T \psi\|_{\mathbf{B}} \leq K\|\varphi-\psi\|_{\mathbf{B}},
$$

which implies that the mapping $T: \mathbf{B} \longrightarrow \mathbf{B}$ is a contraction mapping. Therefore, the mapping $T$ possesses a unique fixed point

$$
x^{* *}=\left(x_{1}^{* *}(t), x_{2}^{* *}(t), \cdots, x_{n}^{* *}(t)\right)^{T} \in \mathbf{B}, T x^{* *}=x^{* *} .
$$


By (3.7) and (3.9), $x^{* *}$ satisfies (3.7). So (1.1) has a unique continuously differentiable pseudo almost periodic solution $x^{*}=\left(\xi_{1} x_{1}^{* *}(t), \xi_{2} x_{2}^{* *}(t), \cdots, \xi_{n} x_{n}^{* *}(t)\right)^{T}$. The proof of Theorem 3.1 is now completed.

\section{Exponential stability of the pseudo almost periodic solution}

In this section, we will discuss the global exponential stability of the pseudo almost periodic solution of system (1.1).

Theorem 4.1. Suppose that all conditions in Theorem 3.1 are satisfied. Moreover, assume that

$$
\begin{aligned}
& \left(1+\frac{c_{i}^{+}}{c_{i}^{-}}\right)\left(c_{i}^{+} \eta_{i}^{+}+\xi_{i}^{-1} \sum_{j=1}^{n} a_{i j}^{+} L_{j}^{f} \xi_{j}+\xi_{i}^{-1} \sum_{j=1}^{n} \sum_{l=1}^{n} b_{i j l}^{+}\left(M_{j}^{g} L_{l}^{g} \xi_{l}+M_{l}^{g} L_{j}^{g} \xi_{j}\right)\right. \\
& +\xi_{i}^{-1} \sum_{j=1}^{n} \sum_{l=1}^{n} d_{i j l}^{+} \int_{0}^{\infty}\left|\sigma_{i j l}(u)\right| d u \int_{0}^{\infty}\left|\nu_{i j l}(u)\right| d u\left(M_{j}^{g} L_{l}^{g} \xi_{l}+M_{l}^{g} L_{j}^{g} \xi_{j}\right) \\
& <1, \quad i \in J .
\end{aligned}
$$

Then system (1.1) has at least one pseudo almost periodic solution $x^{*}(t)$, and $x^{*}(t)$ is globally exponentially stable.

Proof. By Theorem 3.1, (1.1) has a unique continuously differentiable almost periodic solution $x^{*}(t)=\left(x_{1}^{*}(t), x_{2}^{*}(t), \cdots, x_{n}^{*}(t)\right)^{T}$. Suppose that $x(t)=\left(x_{1}(t), x_{2}(t), \cdots, x_{n}(t)\right)^{T}$ is an arbitrary solution of $(1.1)$ associated with initial value $\varphi(t)=\left(\varphi_{1}(t), \varphi_{2}(t), \cdots, \varphi_{n}(t)\right)^{T}$ satisfying (1.2).

Let

$$
\begin{aligned}
y(t) & =\left(y_{1}(t), y_{2}(t), \cdots, y_{n}(t)\right)^{T} \\
& =\left(\xi_{1}^{-1}\left(x_{1}(t)-x_{1}^{*}(t)\right), \xi_{2}^{-1}\left(x_{2}(t)-x_{2}^{*}(t)\right), \cdots, \xi_{n}^{-1}\left(x_{n}(t)-x_{n}^{*}(t)\right)\right)^{T} .
\end{aligned}
$$

Then

$$
\begin{aligned}
y_{i}^{\prime}(t)= & -c_{i}(t) y_{i}\left(t-\eta_{i}(t)\right)+\xi_{i}^{-1} \sum_{j=1}^{n} a_{i j}(t)\left(f_{j}\left(x_{j}\left(t-\tau_{i j}(t)\right)\right)-f_{j}\left(x_{j}^{*}\left(t-\tau_{i j}(t)\right)\right)\right) \\
& +\xi_{i}^{-1} \sum_{j=1}^{n} \sum_{l=1}^{n} b_{i j l}(t)\left(g_{j}\left(x_{j}\left(t-\alpha_{i j l}(t)\right)\right) g_{l}\left(x_{l}\left(t-\beta_{i j l}(t)\right)\right)\right. \\
& \left.-g_{j}\left(x_{j}^{*}\left(t-\alpha_{i j l}(t)\right)\right) g_{l}\left(x_{l}^{*}\left(t-\beta_{i j l}(t)\right)\right)\right) \\
& +\xi_{i}^{-1} \sum_{j=1}^{n} \sum_{l=1}^{n} d_{i j l}(t)\left(\int_{0}^{\infty} \sigma_{i j l}(u) h_{j}\left(x_{j}(t-u)\right) d u \int_{0}^{\infty} \nu_{i j l}(u) h_{l}\left(x_{l}(t-u)\right) d u\right. \\
& \left.-\int_{0}^{\infty} \sigma_{i j l}(u) h_{j}\left(x_{j}^{*}(t-u)\right) d u \int_{0}^{\infty} \nu_{i j l}(u) h_{l}\left(x_{l}^{*}(t-u)\right) d u\right),
\end{aligned}
$$

where $i=1,2, \cdots, n$. 
We can choose a constant $\lambda \in\left(0, \min \left\{\kappa, \min _{i \in J} c_{i}^{-}\right\}\right)$such that

$$
\begin{aligned}
\Gamma_{i}(\lambda)= & -c_{i}^{-}+\lambda+c_{i}^{+} \eta_{i}^{+} e^{\lambda \eta_{i}^{+}}+\xi_{i}^{-1} \sum_{j=1}^{n} a_{i j}^{+} L_{j}^{f} \xi_{j} e^{\lambda \tau_{i j}^{+}} \\
& +\xi_{i}^{-1} \sum_{j=1}^{n} \sum_{l=1}^{n} b_{i j l}^{+}\left[M_{j}^{g} L_{l}^{g} \xi_{l} e^{\lambda \beta_{i j l}^{+}}+M_{l}^{g} L_{j}^{g} \xi_{j} e^{\left.\lambda \alpha_{i j l}^{+}\right]}\right. \\
& +\xi_{i}^{-1} \sum_{j=1}^{n} \sum_{l=1}^{n} d_{i j l}^{+}\left[\int_{0}^{\infty}\left|\sigma_{i j l}(u)\right| d u M_{j}^{h} \int_{0}^{\infty}\left|\nu_{i j l}(u)\right| L_{l}^{h} \xi_{l} e^{\lambda u} d u\right. \\
& +\int_{0}^{\infty}\left|\sigma_{i j l}(u)\right| L_{j}^{h} \xi_{j} e^{\lambda u} d u \int_{0}^{\infty}\left|\nu_{i j l}(u)\right| d u M_{l}^{h} \\
= & \left(c_{i}^{-}-\lambda\right)\left(\frac{\beta_{i}}{c_{i}^{-}-\lambda}-1\right) \\
< & 0,
\end{aligned}
$$

and

$$
\begin{aligned}
\Pi_{i}(\lambda)= & \left(1+\frac{c_{i}^{+}}{c_{i}^{-}-\lambda}\right)\left[c_{i}^{+} \eta_{i}^{+} e^{\lambda \eta_{i}^{+}}+\xi_{i}^{-1} \sum_{j=1}^{n} a_{i j}^{+} L_{j}^{f} \xi_{j} e^{\lambda \tau_{i j}^{+}}\right. \\
& +\xi_{i}^{-1} \sum_{j=1}^{n} \sum_{l=1}^{n} b_{i j l}^{+}\left[M_{j}^{g} L_{l}^{g} \xi_{l} e^{\lambda \beta_{i j l}^{+}}+M_{l}^{g} L_{j}^{g} \xi_{j} e^{\left.\lambda \alpha_{i j l}^{+}\right]}\right. \\
& +\xi_{i}^{-1} \sum_{j=1}^{n} \sum_{l=1}^{n} d_{i j l}^{+}\left[\int_{0}^{\infty}\left|\sigma_{i j l}(u)\right| d u M_{j}^{h} \int_{0}^{\infty}\left|\nu_{i j l}(u)\right| L_{l}^{h} \xi_{l} e^{\lambda u} d u\right. \\
& \left.+\int_{0}^{\infty}\left|\sigma_{i j l}(u)\right| L_{j}^{h} \xi_{j} e^{\lambda u} d u \int_{0}^{\infty}\left|\nu_{i j l}(u)\right| d u M_{l}^{h}\right] \\
= & \left(1+\frac{c_{i}^{+}}{c_{i}^{-}-\lambda}\right) \beta_{i} \\
< & 1,
\end{aligned}
$$

where

$$
\begin{aligned}
\beta_{i}= & c_{i}^{+} \eta_{i}^{+} e^{\lambda \eta_{i}^{+}}+\xi_{i}^{-1} \sum_{j=1}^{n} a_{i j}^{+} L_{j}^{f} \xi_{j} e^{\lambda \tau_{i j}^{+}} \\
& +\xi_{i}^{-1} \sum_{j=1}^{n} \sum_{l=1}^{n} b_{i j l}^{+}\left[M_{j}^{g} L_{l}^{g} \xi_{l} e^{\lambda \beta_{i j l}^{+}}+M_{l}^{g} L_{j}^{g} \xi_{j} e^{\left.\lambda \alpha_{i j l}^{+}\right]}\right. \\
& +\xi_{i}^{-1} \sum_{j=1}^{n} \sum_{l=1}^{n} d_{i j l}^{+}\left[\int_{0}^{\infty}\left|\sigma_{i j l}(u)\right| d u M_{j}^{h} \int_{0}^{\infty}\left|\nu_{i j l}(u)\right| L_{l}^{h} \xi_{l} e^{\lambda u} d u\right. \\
& +\int_{0}^{\infty}\left|\sigma_{i j l}(u)\right| L_{j}^{h} \xi_{j} e^{\lambda u} d u \int_{0}^{\infty}\left|\nu_{i j l}(u)\right| d u M_{l}^{h}, i=1,2, \cdots, n .
\end{aligned}
$$


Let

$$
\left\|\varphi-x^{*}\right\|_{\xi}=\max \left\{\sup _{t \leq 0} \max _{1 \leq i \leq n} \xi_{i}^{-1}\left|\varphi_{i}(t)-x_{i}^{*}(t)\right|, \sup _{t \leq 0} \max _{1 \leq i \leq n} \xi_{i}^{-1}\left|\varphi_{i}^{\prime}(t)-x_{i}^{*}(t)\right|\right\}
$$

and $M$ be a constant such that

$$
M>\frac{c_{i}^{-}-\lambda}{\beta_{i}}>1, \quad \text { for all } i=1,2, \ldots, n,
$$

which, together with (4.3), yields

$$
\frac{1}{M}-\frac{\beta_{i}}{c_{i}^{-}-\lambda}<0, \frac{\beta_{i}}{c_{i}^{-}-\lambda}<1, \quad \text { for all } i \in J
$$

Consequently, for any $\varepsilon>0$, it is obvious that

$$
\|y(t)\|_{1}<\left(\left\|\varphi-x^{*}\right\|_{\xi}+\varepsilon\right) e^{-\lambda t}<M\left(\left\|\varphi-x^{*}\right\|_{\xi}+\varepsilon\right) e^{-\lambda t} \text { for all } t \in(-\infty, 0] \text {. }
$$

In the following, we will show

$$
\|y(t)\|_{1}<M\left(\left\|\varphi-x^{*}\right\|_{\xi}+\varepsilon\right) e^{-\lambda t} \text { for all } t>0 .
$$

Otherwise, there must exist $i \in\{1,2, \cdots, n\}$ and $\theta>0$ such that

$$
\left\{\begin{aligned}
\|y(\theta)\|_{1} & =\max \left\{\left|y_{i}(\theta)\right|,\left|y_{i}^{\prime}(\theta)\right|\right\}=M\left(\left\|\varphi-x^{*}\right\|_{\xi}+\varepsilon\right) e^{-\lambda \theta} \\
\|y(t)\|_{1} & <M\left(\left\|\varphi-x^{*}\right\|_{\xi}+\varepsilon\right) e^{-\lambda t} \text { for all } t \in(-\infty, \theta) .
\end{aligned}\right.
$$

Note that

$$
\begin{aligned}
& y_{i}^{\prime}(s)+c_{i}(s) y_{i}(s) \\
= & c_{i}(s) \int_{s-\eta_{i}(s)}^{s} y_{i}^{\prime}(u) d u \\
& +\xi_{i}^{-1} \sum_{j=1}^{n} a_{i j}(s)\left(f_{j}\left(x_{j}\left(s-\tau_{i j}(s)\right)\right)-f_{j}\left(x_{j}^{*}\left(s-\tau_{i j}(s)\right)\right)\right) \\
& +\xi_{i}^{-1} \sum_{j=1}^{n} \sum_{l=1}^{n} b_{i j l}(s)\left(g_{j}\left(x_{j}\left(s-\alpha_{i j l}(s)\right)\right) g_{l}\left(x_{l}\left(s-\beta_{i j l}(s)\right)\right)\right. \\
& \left.-g_{j}\left(x_{j}^{*}\left(s-\alpha_{i j l}(s)\right)\right) g_{l}\left(x_{l}^{*}\left(s-\beta_{i j l}(s)\right)\right)\right) \\
& +\xi_{i}^{-1} \sum_{j=1}^{n} \sum_{l=1}^{n} d_{i j l}(s)\left(\int_{0}^{\infty} \sigma_{i j l}(u) h_{j}\left(x_{j}(s-u)\right) d u \int_{0}^{\infty} \nu_{i j l}(u) h_{l}\left(x_{l}(s-u)\right) d u\right. \\
& \left.-\int_{0}^{\infty} \sigma_{i j l}(u) h_{j}\left(x_{j}^{*}(s-u)\right) d u \int_{0}^{\infty} \nu_{i j l}(u) h_{l}\left(x_{l}^{*}(s-u)\right) d u\right), s \in[0, t], t \in[0, \theta] .\left(x^{\prime}\right)
\end{aligned}
$$

Multiplying both sides of (4.10) by $e^{\int_{0}^{s} c_{i}(u) d u}$, and integrating it on $[0, t]$, we get

$$
\begin{aligned}
y_{i}(t)= & y_{i}(0) e^{-\int_{0}^{t} c_{i}(u) d u}+\int_{0}^{t} e^{-\int_{s}^{t} c_{i}(u) d u}\left[c_{i}(s) \int_{s-\eta_{i}(s)}^{s} y_{i}^{\prime}(u) d u\right. \\
& +\xi_{i}^{-1} \sum_{j=1}^{n} a_{i j}(s)\left(f_{j}\left(x_{j}\left(s-\tau_{i j}(s)\right)\right)-f_{j}\left(x_{j}^{*}\left(s-\tau_{i j}(s)\right)\right)\right)
\end{aligned}
$$




$$
\begin{aligned}
& +\xi_{i}^{-1} \sum_{j=1}^{n} \sum_{l=1}^{n} b_{i j l}(s)\left(g_{j}\left(x_{j}\left(s-\alpha_{i j l}(s)\right)\right) g_{l}\left(x_{l}\left(s-\beta_{i j l}(s)\right)\right)\right. \\
& \left.-g_{j}\left(x_{j}^{*}\left(s-\alpha_{i j l}(s)\right)\right) g_{l}\left(x_{l}^{*}\left(s-\beta_{i j l}(s)\right)\right)\right) \\
& +\sum_{j=1}^{n} \sum_{l=1}^{n} d_{i j l}(s)\left(\int_{0}^{\infty} \sigma_{i j l}(u) h_{j}\left(x_{j}(s-u)\right) d u \int_{0}^{\infty} \nu_{i j l}(u) h_{l}\left(x_{l}(s-u)\right) d u\right. \\
& \left.\left.-\int_{0}^{\infty} \sigma_{i j l}(u) h_{j}\left(x_{j}^{*}(s-u)\right) d u \int_{0}^{\infty} \nu_{i j l}(u) h_{l}\left(x_{l}^{*}(s-u)\right) d u\right)\right] d s, t \in[0, \theta] .
\end{aligned}
$$

Thus, with the help of (4.7), we have

$$
\begin{aligned}
\left|y_{i}(\theta)\right|= & \mid y_{i}(0) e^{-\int_{0}^{\theta} c_{i}(u) d u}+\int_{0}^{\theta} e^{-\int_{s}^{\theta} c_{i}(u) d u}\left[c_{i}(s) \int_{s-\eta_{i}(s)}^{s} y_{i}^{\prime}(u) d u\right. \\
& +\xi_{i}^{-1} \sum_{j=1}^{n} a_{i j}(s)\left(f_{j}\left(x_{j}\left(s-\tau_{i j}(s)\right)\right)-f_{j}\left(x_{j}^{*}\left(s-\tau_{i j}(s)\right)\right)\right) \\
& +\xi_{i}^{-1} \sum_{j=1}^{n} \sum_{l=1}^{n} b_{i j l}(s)\left(g_{j}\left(x_{j}\left(s-\alpha_{i j l}(s)\right)\right) g_{l}\left(x_{l}\left(s-\beta_{i j l}(s)\right)\right)\right. \\
& \left.-g_{j}\left(x_{j}^{*}\left(s-\alpha_{i j l}(s)\right)\right) g_{l}\left(x_{l}^{*}\left(s-\beta_{i j l}(s)\right)\right)\right) \\
& +\xi_{i}^{-1} \sum_{j=1}^{n} \sum_{l=1}^{n} d_{i j l}(s)\left(\int_{0}^{\infty} \sigma_{i j l}(u) h_{j}\left(x_{j}(s-u)\right) d u \int_{0}^{\infty} \nu_{i j l}(u) h_{l}\left(x_{l}(s-u)\right) d u\right. \\
& \left.\left.-\int_{0}^{\infty} \sigma_{i j l}(u) h_{j}\left(x_{j}^{*}(s-u)\right) d u \int_{0}^{\infty} \nu_{i j l}(u) h_{l}\left(x_{l}^{*}(s-u)\right) d u\right)\right] d s \mid \\
\leq & \left(\left\|\varphi-x^{*}\right\|_{\xi}+\varepsilon\right) e^{-c_{i}^{-} \theta}+\int_{0}^{\theta} e^{-\int_{s}^{\theta} c_{i}(u) d u}\left[c_{i}(s) \eta_{i}(s) M\left(\left\|\varphi-x^{*}\right\|_{\xi}+\varepsilon\right) e^{-\lambda\left(s-\eta_{i}(s)\right)}\right. \\
& +\xi_{i}^{-1} \sum_{j=1}^{n} a_{i j}^{+} L_{j}^{f} \xi_{j} M\left(\left\|\varphi-x^{*}\right\|_{\xi}+\varepsilon\right) e^{-\lambda\left(s-\tau_{i j}(s)\right)} \\
& \left.+\int_{0}^{\infty}\left|\sigma_{i j l}(u)\right| L_{j}^{h} \xi_{j} M\left(\left\|\varphi-x^{*}\right\|_{\xi}+\varepsilon\right) e^{-\lambda(s-u)} d u \int_{0}^{\infty}\left|\nu_{i j l}(u)\right| d u M_{l}^{h}\right] d s \\
& +\xi_{i}^{-1} \sum_{j=1}^{n} \sum_{l=1}^{n} b_{i j l}^{+}\left[M_{j}^{g} L_{l}^{g} \xi_{l} M\left(\left\|\varphi-x^{*}\right\|_{\xi}+\varepsilon\right) e^{-\lambda\left(s-\beta_{i j l}(s)\right)}\right. \\
& \left.+M_{l}^{g} L_{j}^{g} \xi_{j} M\left(\left\|\varphi-x^{*}\right\|_{\xi}+\varepsilon\right) e^{-\lambda\left(s-\alpha_{i j l}(s)\right)}\right] \\
& +\xi_{i}^{-1} \sum_{j=1}^{n} \sum_{l=1}^{n} d_{i j l}^{+}\left[\int_{0}^{\infty}\left|\sigma_{i j l}(u)\right| d u M_{j}^{h} \int_{\xi}^{\infty}\left|\nu_{i j l}(u)\right| L_{l}^{h} \xi_{l} M\left(\left\|\varphi-x^{*}\right\|_{\xi}+\varepsilon\right) e^{-\lambda(s-u)} d u\right. \\
& \\
& \\
& \\
& \\
&
\end{aligned}
$$




$$
\begin{aligned}
& +\xi_{i}^{-1} \sum_{j=1}^{n} a_{i j}^{+} L_{j}^{f} \xi_{j} e^{\lambda \tau_{i j}^{+}} \\
& +\xi_{i}^{-1} \sum_{j=1}^{n} \sum_{l=1}^{n} b_{i j l}^{+}\left[M_{j}^{g} L_{l}^{g} \xi_{l} e^{\lambda \beta_{i j l}^{+}}+M_{l}^{g} L_{j}^{g} \xi_{j} e^{\left.\lambda \alpha_{i j l}^{+}\right]}\right. \\
& +\xi_{i}^{-1} \sum_{j=1}^{n} \sum_{l=1}^{n} d_{i j l}^{+}\left[\int_{0}^{\infty}\left|\sigma_{i j l}(u)\right| d u M_{j}^{h} \int_{0}^{\infty}\left|\nu_{i j l}(u)\right| L_{l}^{h} \xi_{l} e^{\lambda u} d u\right. \\
& \left.+\int_{0}^{\infty}\left|\sigma_{i j l}(u)\right| L_{j}^{h} \xi_{j} e^{\lambda u} d u \int_{0}^{\infty}\left|\nu_{i j l}(u)\right| d u M_{l}^{h}\right] d s M\left(\left\|\varphi-x^{*}\right\|_{\xi}+\varepsilon\right) \\
\leq & \left(\left\|\varphi-x^{*}\right\|_{\xi}+\varepsilon\right) e^{-c_{i}^{-} \theta}+e^{-c_{i}^{-} \theta} \int_{0}^{\theta} e^{\left(c_{i}^{-}-\lambda\right) s} d s \beta_{i} M\left(\left\|\varphi-x^{*}\right\|_{\xi}+\varepsilon\right) \\
= & M\left(\left\|\varphi-x^{*}\right\|_{\xi}+\varepsilon\right) e^{-\lambda \theta}\left[\left(\frac{1}{M}-\frac{\beta_{i}}{c_{i}^{-}-\lambda}\right) e^{\left(\lambda-c_{i}^{-}\right) \theta}+\frac{\beta_{i}}{c_{i}^{-}-\lambda}\right] \\
< & M\left(\left\|\varphi-x^{*}\right\|_{\xi}+\varepsilon\right) e^{-\lambda \theta},
\end{aligned}
$$

which, together with (4.9), implies that

$$
\|y(\theta)\|_{1}=\max \left\{\left|y_{i}(\theta)\right|,\left|y_{i}^{\prime}(\theta)\right|\right\}=\left|y_{i}^{\prime}(\theta)\right|=M\left(\left\|\varphi-x^{*}\right\|_{\xi}+\varepsilon\right) e^{-\lambda \theta} .
$$

From (4.4) and (4.7), (4.10) and (4.11) yield

$$
\begin{aligned}
\left|y_{i}^{\prime}(\theta)\right| \leq & \left|c_{i}(\theta) y_{i}(\theta)\right|+c_{i}(\theta) \int_{\theta-\eta_{i}(\theta)}^{\theta}\left|y_{i}^{\prime}(u)\right| d u \\
& +\xi_{i}^{-1} \sum_{j=1}^{n}\left|a_{i j}(\theta)\right|\left|f_{j}\left(x_{j}\left(\theta-\tau_{i j}(\theta)\right)\right)-f_{j}\left(x_{j}^{*}\left(\theta-\tau_{i j}(\theta)\right)\right)\right| \\
& +\xi_{i}^{-1} \sum_{j=1}^{n} \sum_{l=1}^{n}\left|b_{i j l}(\theta)\right|\left[\mid g_{j}\left(x_{j}\left(\theta-\alpha_{i j l}(\theta)\right)\right) g_{l}\left(x_{l}\left(\theta-\beta_{i j l}(\theta)\right)\right)\right. \\
& -g_{j}\left(x_{j}\left(\theta-\alpha_{i j l}(\theta)\right)\right) g_{l}\left(x_{l}^{*}\left(\theta-\beta_{i j l}(\theta)\right)\right) \mid \\
& +\mid g_{j}\left(x_{j}\left(\theta-\alpha_{i j l}(\theta)\right)\right) g_{l}\left(x_{l}^{*}\left(\theta-\beta_{i j l}(\theta)\right)\right) \\
& \left.-g_{j}\left(x_{j}^{*}\left(\theta-\alpha_{i j l}(\theta)\right)\right) g_{l}\left(x_{l}^{*}\left(\theta-\beta_{i j l}(\theta)\right)\right) \mid\right] \\
& +\xi_{i}^{-1} \sum_{j=1}^{n} \sum_{l=1}^{n}\left|d_{i j l}(\theta)\right|\left[\mid \int_{0}^{\infty} \sigma_{i j l}(u) h_{j}\left(x_{j}(\theta-u)\right) d u \int_{0}^{\infty} \nu_{i j l}(u) h_{l}\left(x_{l}(\theta-u)\right) d u\right. \\
& -\int_{0}^{\infty} \sigma_{i j l}(u) h_{j}\left(x_{j}(\theta-u)\right) d u \int_{0}^{\infty} \nu_{i j l}(u) h_{l}\left(x_{l}^{*}(\theta-u)\right) d u \mid \\
& +\mid \int_{0}^{\infty} \sigma_{i j l}(u) h_{j}\left(x_{j}(\theta-u)\right) d u \int_{0}^{\infty} \nu_{i j l}(u) h_{l}\left(x_{l}^{*}(\theta-u)\right) d u \\
& \left.-\int_{0}^{\infty} \sigma_{i j l}(u) h_{j}\left(x_{j}^{*}(\theta-u)\right) d u \int_{0}^{\infty} \nu_{i j l}(u) h_{l}\left(x_{l}^{*}(\theta-u)\right) d u \mid\right]
\end{aligned}
$$




$$
\begin{aligned}
& \leq M\left(\left\|\varphi-x^{*}\right\|_{\xi}+\varepsilon\right) e^{-\lambda \theta}\left[c_{i}^{+}\left(\frac{1}{M}-\frac{\beta_{i}}{c_{i}^{-}-\lambda}\right) e^{\left(\lambda-c_{i}^{-}\right) \theta}+\beta_{i}\left(\frac{c_{i}^{+}}{c_{i}^{-}-\lambda}+1\right)\right] \\
& <M\left(\left\|\varphi-x^{*}\right\|_{\xi}+\varepsilon\right) e^{-\lambda \theta}
\end{aligned}
$$

which contradicts (4.13). Hence, (4.8) holds. Letting $\varepsilon \longrightarrow 0^{+}$, we have from (4.8) that

which implies

$$
\|y(t)\|_{1} \leq M\left\|\varphi-x^{*}\right\|_{\xi} e^{-\lambda t} \text { for all } t>0
$$

$$
\left\|x(t)-x^{*}(t)\right\|_{1} \leq M\left\|\varphi-x^{*}\right\|_{0} e^{-\lambda t} \text { for all } t>0 .
$$

This completes the proof.

\section{Example and Remark}

In this section, some examples and remarks are provided to demonstrate the effectiveness of our results.

Example 5.1. Consider the following for HCNNs with time-varying leakage delays:

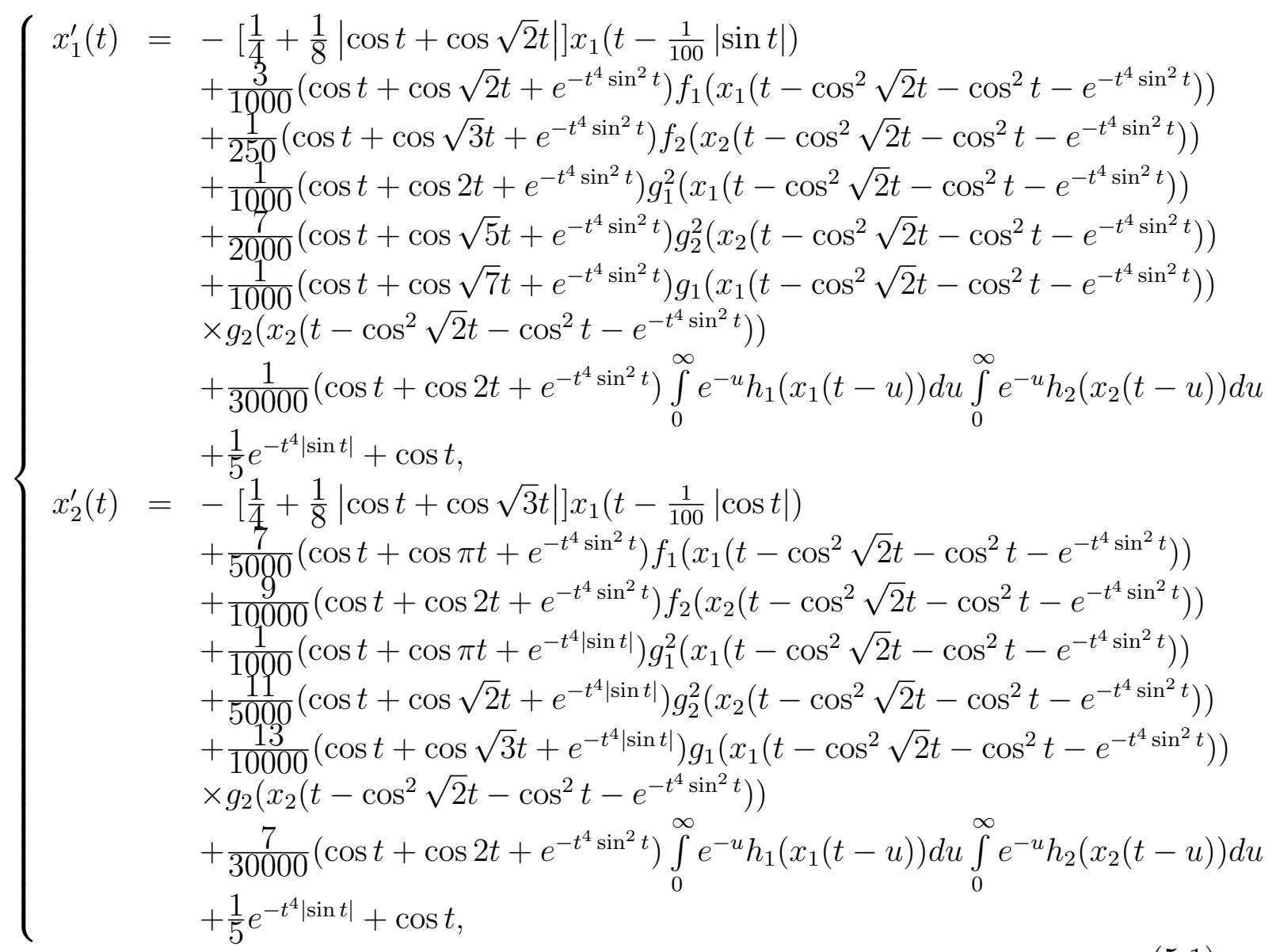

where

$$
f_{j}(x)=\frac{1}{4}(|x|+\cos x), g_{j}(x)=h_{j}(x)=\cos x
$$




$$
b_{i j l}(u)=0,(i j l \neq 112, i j l \neq 212), \sigma_{i j l}(u)=v_{i j l}(u)=e^{-u}, i, j, l=1,2 .
$$

Comparing (5.1) with (1.1) and using some basic information, it follows that

$$
\begin{gathered}
c_{1}^{-}=\frac{1}{4}, c_{1}^{+}=\frac{1}{2}, c_{2}^{-}=\frac{1}{4}, c_{2}^{+}=\frac{1}{2}, \eta_{j}^{+}=\frac{1}{100}, L_{j}^{f}=\frac{1}{2} \\
L_{j}^{g}=L_{j}^{h}=L_{l}^{h}=1, M_{j}^{g}=M_{j}^{h}=M_{l}^{h}=1, \xi_{i}=\xi_{i}^{-1}=\xi_{k}=\xi_{l}=1 \\
-c_{i}^{-}+c_{i}^{+} \eta_{i}^{+}+\xi_{i}^{-1} \sum_{j=1}^{2} a_{i j}^{+} L_{j}^{f} \xi_{i}+\xi_{i}^{-1} \sum_{j=1}^{2} \sum_{l=1}^{2} b_{i j l}^{+}\left(M_{j}^{g} L_{l}^{g} \xi_{l}+M_{l}^{g} L_{j}^{g} \xi_{j}\right), \\
+\xi_{i}^{-1} \sum_{j=1}^{2} \sum_{l=1}^{2} d_{i j l}^{+} \int_{0}^{\infty}\left|\sigma_{i j l}(u)\right| d u \int_{0}^{\infty}\left|v_{i j l}(t)\right| d u\left(M_{j}^{h} L_{l}^{h} \xi_{l}+M_{l}^{h} L_{j}^{h} \xi_{j}\right), \\
=-\frac{1}{4}+\frac{1}{200}+\left(\frac{9}{2000}+\frac{3}{500}+\frac{21}{10000}+\frac{27}{2000}\right)+\left(\frac{12}{1000}+\frac{156}{10000}\right) \\
+\frac{8}{10000}\left(\int_{0}^{\infty} e^{-u} d u \int_{0}^{\infty} e^{-u} d u\right)=-\frac{2181}{10000}=-\alpha_{i}, \\
c_{i}^{-}-\alpha_{i}+c_{i}^{+}\left(1-\frac{\alpha_{i}}{c_{i}^{+}}\right)=\frac{1569}{2500}<1
\end{gathered}
$$

and

$$
\begin{aligned}
& \left(1+\frac{c_{i}^{+}}{c_{i}^{-}}\right)\left(c_{i}^{+} \eta_{i}^{+}+\xi_{i}^{-1} \sum_{j=1}^{2} a_{i j}^{+} L_{j}^{f} \xi_{i}+\xi_{i}^{-1} \sum_{j=1}^{2} \sum_{l=1}^{2} b_{i j l}^{+}\left(M_{j}^{g} L_{l}^{g} \xi_{l}+M_{l}^{g} L_{j}^{g} \xi_{j}\right),\right. \\
& \quad+\xi_{i}^{-1} \sum_{j=1}^{2} \sum_{l=1}^{2} d_{i j l}^{+} \int_{0}^{\infty}\left|\sigma_{i j l}(u)\right| d u \int_{0}^{\infty}\left|v_{i j l}(t)\right| d u\left(M_{j}^{h} L_{l}^{h} \xi_{l}+M_{l}^{h} L_{j}^{h} \xi_{j}\right) \\
& \left.\quad=3\left[\frac{1}{200}+\left(\frac{261}{10000}\right)\right]+\frac{8}{10000}\left(\int_{0}^{\infty} e^{-u} d u \int_{0}^{\infty} e^{-u} d u\right)\right]=\frac{957}{10000}<1,
\end{aligned}
$$

which imply that (5.1) satisfies all the conditions in Theorem 3.1 and Theorem 4.1. Hence, we can conclude that system (5.1) has a unique continuously differentiable pseudo almost periodic solution $x^{*}(t)$, which is globally exponentially stable with the exponential convergent rate $\lambda \approx 0.001$. 


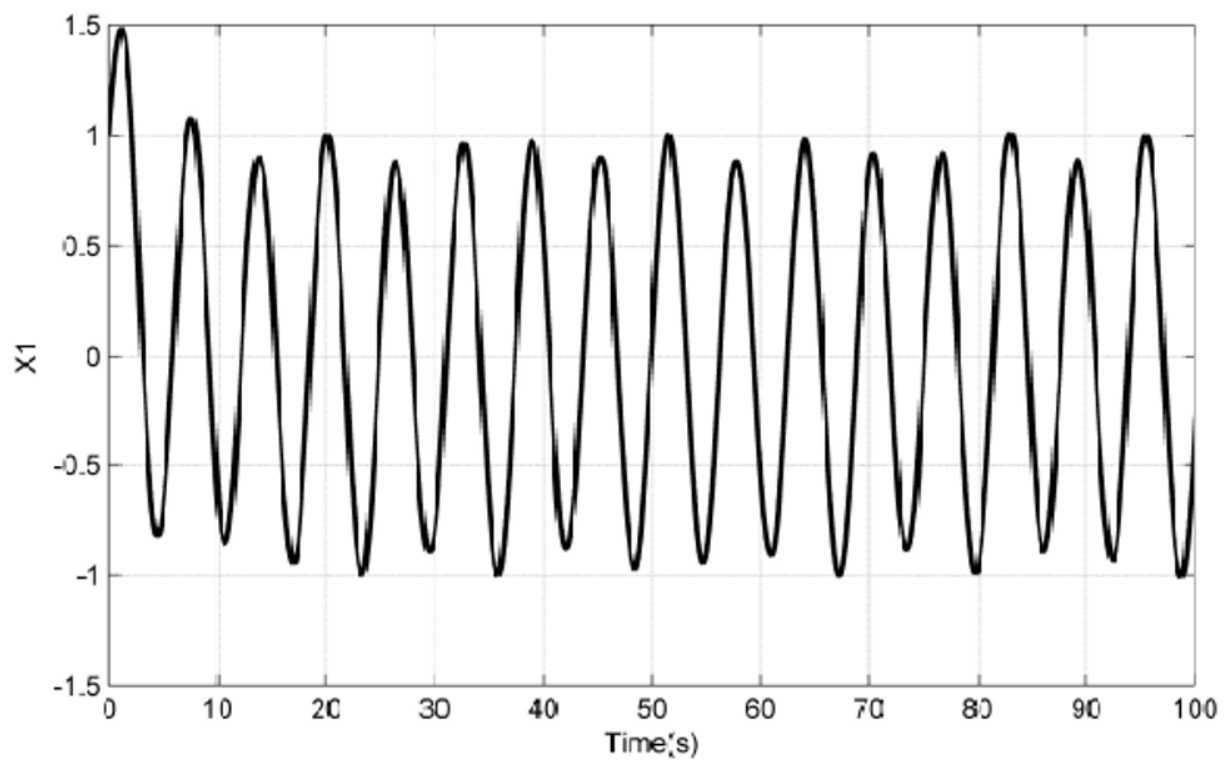

Figure 1: Trajectories $x_{1}(t)$ of 5.1 in Example 5.1 with $\tau=0,25$

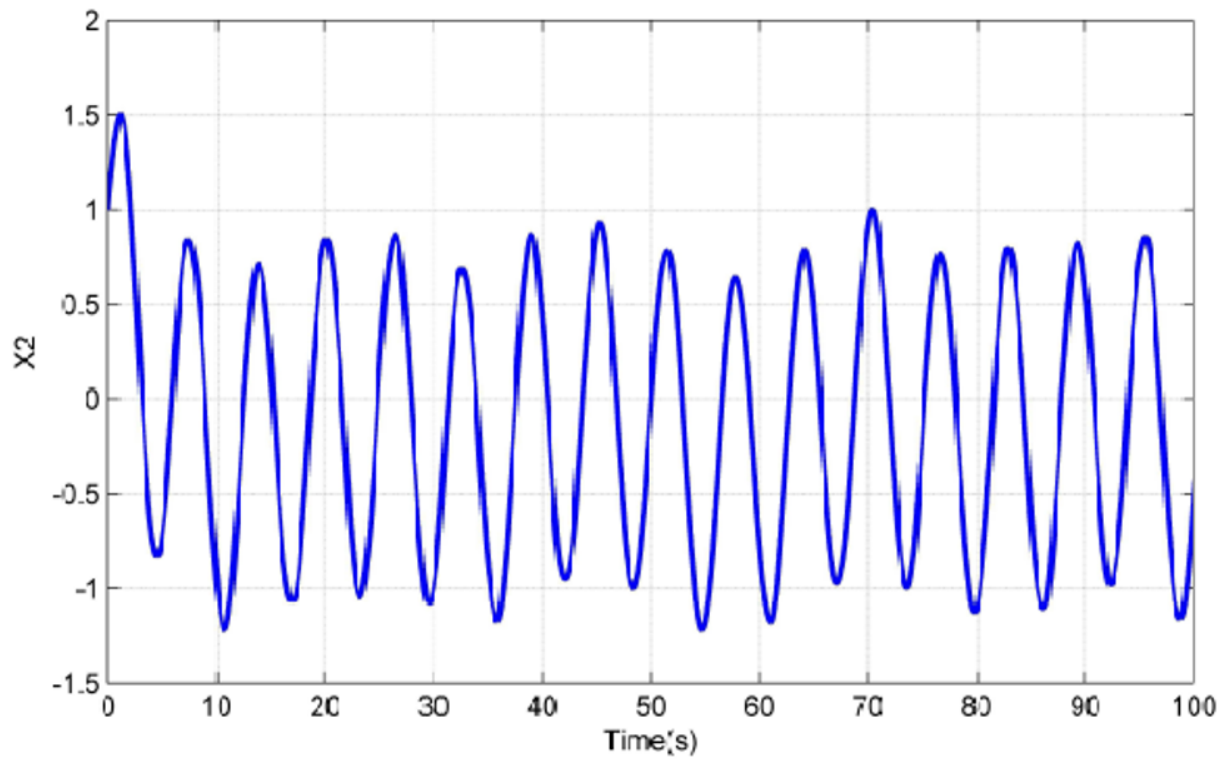

Figure 2: Trajectories $x_{2}(t)$ of 5.1 in Example 5.1 with $\tau=0,25$

Open Access: This article is distributed under the terms of the Creative Commons Attribution License (CC-BY 4.0) which permits any use, distribution, and reproduction in any medium, provided the original author(s) and the source are credited. 


\section{References}

[1] A. Dembo, O. Farotimi, T. Kailath, High-order absolutely stable neural networks. IEEE Trans. Circuits Syst. 38 (1991) 57-65.

[2] D. Psaltis, C. H. Park, J. Hong, Higher order associative memories and their optical implementations. Neural Networks 1 (1988), 143-163.

[3] N. B. Karayiannis, A. N. Venetsanopoulos, On the training and performance of high-order neural networks. Math. Biosci. 129 (1995), no. 2, 143-168.

[4] Z. Chen, M. Yang, Exponential convergence for HRNNs with continuously distributed delays in the leakage terms. Neural Comput \& Applic (2013) 23:2221-2229.

[5] R. Jia, M. Yang, Convergence for HRNNs with Unbounded Activation Functions and Timevarying Delays in the Leakage Terms. Neural Process Lett (2014) 39: 69-79.

[6] Y. Yu, W. Jiao, New Results on Exponential Convergence for HRNNs with Continuously Distributed Delays in the Leakage Terms. Neural Process Lett (2014) 39:167-177.

[7] Y. Xu, Anti-periodic solutions for HCNNs with time-varying delays in the leakage terms, Neural Comput \& Applic (2012) DOI 10.1007/s00521-012-1330-6.

[8] A. Zhang, Existence and exponential stability of anti-periodic solutions for HCNNs with timevarying leakage delays. Adv. Difference Equ. 2013, 2013:162, 14 pp.

[9] C. Zhang, Almost Periodic Type Functions and Ergodicity. Kluwer Academic/Science Press, Beijing (2003)

[10] A. M. Fink, Almost periodic differential equations, Lecture Notes in Mathematics, Vol. 377, Springer, Berlin, 1974.

[11] B. Liu, Pseudo Almost Periodic Solutions for CNNs with Continuously Distributed Leakage Delays, Neural Processing Lett. (2014) DOI 10.1007/s11063-014-9354-9.

[12] W. Wang, B. Liu, Global exponential stability of pseudo almost periodic solutions for SICNNs with time-varying leakage delays, Abstr. Appl. Anal. 2014(967328) (2014) 1-17.

[13] B. Liu, Pseudo almost periodic solutions for neutral type CNNs with continuously distributed leakage delays, Neurocomputing 148 (2015) 445-454..

[14] Y. Xu, New results on almost periodic solutions for CNNs with time-varying leakage delays, Neural Computing \& Applications. 25(6)(2014) 1293-1302.

[15] C. Zhang, Pseudo almost periodic solutions of some differential equations II, J. Math. Anal. Appl. , 7-192, 543-561, 1995.

[16] B. Ammar, F. Cherif, A. M. Alimi, Existence and uniqueness of pseudo almost-periodic solutions of recurrent neural networks with time-varying coefficients and mixed delays, IEEE Transactions on Neural Networks and Learning Systems, 23(1) (2012) 109-118. 